\title{
Research on Design and Implementation of Intelligent Home Remotely Web Management and Control Platform
}

\author{
Yan Meng ${ }^{1, a}$
}

Changchun Guanghua University,NO.3555, Wuhan Road, Changchun Economic Development

Zone, Jilin Prov , China, 130000

maomao192210@163.com

Keywords: Design, Implementation, Intelligent Home, Web Management and Control Platform

\begin{abstract}
Intelligent home has greatly changed people's traditional way of life, but at this stage most of its applications are in the user's home and the remote control support slightly is inadequate. For the status quo, using ASP.NET technology and MySQL database development tools to design and implement the intelligent home remote web management and control platform to meet the needs of the user. It not only facilitates the user's use of intelligent home system, but also promotes the development of smart home.
\end{abstract}

\section{Introduction}

With the development of electronic information technology, since 1980, the world appeared electronic home machine, then there is a residential electronic (HE, Home Electronics). Since 1985, the further development of residential electronics, household appliances and communications facilities, security and security facilities to integrate each other, to achieve the residential automation (HA, Home Automation). Since 1988, the further development of electronic information technology, a variety of large companies began the development of residential automation systems, including the earlier commercial home automation system is the United States Smart Home system, the system uses bus technology to achieve the electrical monitoring and management, Become a modern smart home system master and origin.

Although the concept of smart home in the residential automation has long been a long time ago, but in the last century 80's appeared in the residential automation after the use of intelligent home to the specific examples of the building gradually appeared. One of the earliest smart homes in the United States Hartford City, known as the City Place Building, that is, intelligent building, the building was in 1984 by the United States joint technology company building. From the beginning of this building, we should open the world's smart home development prelude.

\section{The Concept of Smart Home}

In recent years, with the improvement of the national economy and scientific and technological level, especially the rapid development of computer technology, network technology, communication technology and control technology, the social informationization has been accelerated, and the relationship between people's work, life and communication has been increasing. Information society in the change of people's work habits and lifestyle at the same time, the traditional residential automation, information technology, environmental comfort, security challenges. Informatization affects people's lives in all aspects, changed people's habits, improve people's quality of life, home intelligence is also in this situation came into being.

Intelligent home system is the use of advanced computer, embedded systems and network communications technology, the family of various devices (such as lighting systems, environmental control, security systems, network appliances) through the home network to connect together. On the one hand, smart home will allow users to have more convenient means to manage the home device, for example, through the wireless remote control, telephone, Internet or voice recognition way to control household equipment; the other hand, intelligent home within a variety of equipment 
Communication, no user control can also be based on different state interaction, it is to improve the quality of life of modern human beings, to create a comfortable, safe and convenient living space has a very important significance.

The development and construction of intelligent home is the inevitable trend of science and technology development in the 21 st century. As the popularity of information technology and application, it greatly promoted the process of home intelligent construction, more intelligent home to provide a reliable technical support, the implementation of more easily and simply. As the smart home system is safe, convenient, efficient, fast, intelligent and personalized unique charm, it has a very broad market prospects, I believe that in the near future will be popular in the ordinary family.

\section{The Intelligent Home Control System}

In smart home applications, the most important indicator is the user experience. This involves many factors and the system control is one of them. Users in the family want to easily control the family within a variety of electrical appliances and equipment. For example, when the user rests on the sofa in the living room, he wants to soften the living room lighting, close the windows, open the air conditioning to adjust the room temperature, turn on the sound to play the light music, all these operations do not need the user to leave the rest of the sofa, on the smart home infrared remote control of a few keys can be achieved. Infrared remote control light portable, is a family member in the home internal control intelligent home an important means.

Users go out from home, especially long-term travel, very concerned about the situation within the home, this time he (she) can log on the Internet, through the web to watch the home video, understand the current situation of the home, modify the smart home security strategy, Home internal equipment to control. These functions are provided by web-based intelligent home remote control system. Remote control systems can be used wherever access is available to the Internet. Web-based intelligent home remote control system in addition to providing video surveillance, security policy development, equipment control functions, due to a simple and flexible web interface, but also in the local system for the provision of powerful configuration and management functions, as a system administrator system configuration tool.

Smart home remote control system by the network, hardware and software to build a combination of web pages as a user interface to operate, there are local operations and remote access to two ways. Local operation does not require intelligent home system access to the internet, used within the family; remote access requires home access to the Internet, in any place where you can use the internet, as long as the open web browser, enter the smart home ip address, you can use The system has security authentication and user management functions to ensure the security of remote access.

In summary, web-based remote control system is an important part of intelligent home system.

\section{The Control Platform Function Analysis}

The intelligent home web management and control platform is designed to display the various environmental parameter information collected by the unified controller located in the user's home to the user and provide the user with remote operation to control the appliance to achieve the function of changing the running status of the appliance. (1) The location of the remote control platform to be processed includes the following categories: (1) the switch status of the various home appliances where the sensor is installed; (2) the installation location of the appliance (5) through the server to the home controller to send control instructions; (6) the number of users in each household electrical information and the number of sensors; (3) the number of hours of electrical appliances; location information.

In order to meet the above requirements, this control platform needs to have the following functions: (1) Two-way communication capability with the remote unified controller according to the instruction of the user, the control platform needs real-time access to various environmental variables and home appliance switching status. (2) the information storage function to obtain a 
variety of electrical operation parameters need to be stored in the database for the query and for other functions to use, the user can switch to the control of the device, (3) real-time display function Users can view and control the home appliance in real time through the web platform, which requires the switch status and other environment variables of the appliance to be displayed in a visual form, and is presented to the user visually and manipulated and controlled by the user. ) Statistical report function system according to the appliance switch time and preset to the database of home appliances and other information on a regular basis to calculate the user's total electricity consumption in the form of statements and charts displayed to the user and the amount of electricity to reach the threshold to remind the user (5) electrical appliances, sensor management users in the family of electrical appliances and sensors in the actual operation is constantly changing, the system should support this dynamic changes, according to different permissions to make changes to the corresponding content.

\section{The Design and Implementation of Management and Control Platform}

The purpose of this platform is to provide users with remote control of home appliances management system. The platform uses. NET and SQL technology, the most suitable for the .NET Framework language $\mathrm{C} \#$ to achieve the realization of the various parts of the platform using different technologies to complete: network communication Using the socket socket programming in $\mathrm{C} \#$, the database uses SQL statements to achieve the establishment of the data structure and the entire database maintenance, visual interface using Javascript and Ajax technology to achieve a variety of specific page effects and no refresh display.

Socket Communication Management. The control platform needs to be in the user's home with the unified control manager to interact, complete the network data communication and then complete the platform of remote control management functions between the network communication between them need to achieve through the Socket communication mechanism to achieve. Socket, that is, "socket" by the IP address and port to describe, it can send requests and data can also receive requests and data, use it can be very convenient to achieve network communication.Socket interface is located in the TCP / IP four-tier model (1) Streaming Sockets (SCOKET_STREAM), streaming sockets are used to provide connection-oriented connectivity. (2) datagram socket (SOCK_DGRAM), the datagram socket provides a connectionless, non-connectionable, and reliable, data transmission service. The service will ensure that the data can be error-free, non-repeatable and received in sequence. The service does not guarantee the reliability of the data transmission, the data may be lost in the transmission process or data duplication, and cannot guarantee the order to receive data. Data packets using UDP (User Datagram protocol) protocol data (SOCK_RAW): The difference between the original socket and the standard socket (the standard socket refers to the stream socket and datagram socket described earlier) is that the original socket The socket can read and write IP packets that are not processed by the kernel, and the stream socket can only read the data of the TCP protocol. The packet socket can only read the UDP protocol data, so if you want to access other protocols the data must use the original socket.

Database Design. The database design follows three principles: the security mechanism of the database, the rationality of the data as a whole, and the reduction of redundant data. The management control system data mainly has the following three categories: the system user's personal information, all kinds of electrical and sensor products information, electrical and sensor products in the operation of the state information in these three types of information, the first two mainly in the system initialization, the user to use the registration, follow up a small amount of changes, the third type of data frequently updated, and provided to the report function module. The use of MySQL to establish a relational database, the database using the number of each number as the main foreign key to link the user's personal information stored in the Userlnfo table, the sensor product information stored in the Prouductlnfo table, electrical information stored in the Equipment lnfo table, electrical operation The status information is stored in the User Equipmentstatetable.

The Design and Implementation of Visual Interface. Visual interface includes user 
registration, login, electrical and sensor delete and modify, electrical and sensor interface, the control of home appliances. Visual interface functions are as follows:

(1) User registration, landing: the user to use the platform to be registered first, fill in the complete personal information, add a unique identity (such as ID number, cell phone number, etc.) to distinguish between the user's electrical and sensor products.

(2) Add and remove electrical and sensor: add complete product information and pictures, intuitively show the product to the user, while providing preset data (such as power) for other functions.

(3) The relationship between electrical and sensor products: the user has a number of electrical appliances and a number of sensors, they should be one-to-one correspondence between the interface will link them so that when the control command to a sensor to the corresponding Electrical equipment to make instructions.

(4) Control function: This function is the core of the platform, into this interface, the system automatically query the family associated with the sensor electrical status, displayed to the user, while providing control options.

\section{Conclusion}

Today, the Internet has changed people's many traditional lifestyles and people can easily and quickly access the Internet. Therefore, it is necessary and timely for the intelligent home system to increase the remote Web management and control platform. This control platform can be very good to meet the user's meet to remote control the changing needs of the family environment, making smart home more secure, comfortable and convenient.

\section{References}

[1] Huifang Zhou: Intelligent home system based on wireless sensor [J]. Microcomputer Information. 2014, 16(3): 62-67.

[2] Hongli Zhang: Intelligent home: the status quo, research and thinking [J]. Electronic Products World. 2014, 5(3): 74-76

[3] Qin Guo: Design of a ZigBee Wireless Sensor Network Node [J]. Automation Technology \& Application.2011, 12(4):11-14

[4] Jieming Liu: Home network specification and standard research [J]. Home Appliances Technology 2010, 7(3):121-124

[5] Jia Xinzhang, Li Jingyuan. Exploration of Intelligent Buildings, computer application 2004, 16(3): $25-26$ 